\title{
Careers in Participant Sport and Other Free Time Activities during Youth and Young Adulthood in South and East Mediterranean Countries
}

\begin{abstract}
By Ken Roberts ${ }^{*}$, Siyka Kovacheva ${ }^{ \pm} \&$ Stanimir Kabaivanov ${ }^{*}$
This paper presents evidence from surveys in 2015-16 of nationally representative samples of approximately 2000 15-29 year olds in each of five South and East Mediterranean countries (Algeria, Egypt, Lebanon, Morocco and Tunisia). The analysis examines the interactive effects of age, gender, family class origins (indicated by fathers' education), and whether respondents progressed through higher education and their current labour market positions, on participation rates in sport plus two social and three cultural free time activities. We find that each free time activity had its own profile in terms of the relationships between participation and the predictor variables, and that sport differed from all the other activities in the width of gender differences, the relatively steep decline in participation from age 15-19 to 20-24 then 25-29, and the strong and enduring influence of family class origins. A conclusion is that in South and East Mediterranean countries (and probably elsewhere), although sport participation may be boosted among adolescents by initiatives in school education and community associations, participation rates will then begin to sink back except when individuals have been reared in households with supportive family free time cultures.
\end{abstract}

Keywords: Arab, free time, North Africa, South and East Mediterranean, sport, young adults, youth.

\section{Introduction}

Background

There have been campaigns to involve more young people throughout the history of modern sports. Only the rationales have changed: to form noble characters, to teach teamwork and leadership, to foster group identity, to train young men for military service, to nurture internationally competitive athletes, and most recently to address an 'obesity crisis' as part of a shift towards healthier modern lifestyles. Only the latter rationale requires young people to be not only recruited but to be retained in sport, not just throughout youth but for their entire subsequent adult lives.

This recent source of interest in young people's sport careers has coincided with a worldwide lengthening of the youth life stage with upward movements in typical ages of completing full-time education, commencing adult employment,

\footnotetext{
*Emeritus Professor of Sociology, University of Liverpool, UK.

${ }^{ \pm}$Associate Professor, University of Plovdiv, Bulgaria.

${ }^{\star}$ Associate Professor, University of Plovdiv, Bulgaria.
} 
and parenthood. During this extended life stage, young people experience short careers though upper secondary and subsequent education and training, in youth jobs, in peer group and romantic relationships, sometimes in housing, always in their leisure lives. Nowadays eleven and 12 year olds in most countries must learn how it is appropriate for teenagers to use social media, and when aged 19 and 20 they must learn to replace 'childish' with new age appropriate uses (Waechter and Hollauf, 2018). In Britain youth careers have been identified in time spent 'hanging about' on neighbourhood streets (MacDonald and Shildrick, 2007), and how as they grow older young people must seek out new appropriate places to go and things to do in their free time (Hendry, 1983; Hendry et al, 1993, 2002).

A series of studies have found that uses of free time in adulthood, even in lateadulthood, often feature skills and tastes that were learnt when young (Bennett, 2006, 2013; Bennett and Hodkinson, 2012; Hodkinson, 2002, 2011; Maguire et al, 1987; Scott and Willitts, 1989). However, discovering that those involved when adults and in later life first became involved when much younger is not the same as showing that most young enthusiasts remain loyal to their tastes and activities. Throughout recent decades UK sport participation rates among children and young people have been high and rising (see, for example, Department for Culture, Media and Sport, 2008; Mason, 1995; Sport England, 2003), but this has not fed through into higher rates of adult participation. Nor have occupational upgrading (more non-manual and fewer manual jobs), or increased participation in higher education, both cross-sectionally related positively to sport participation, boosted overall adult participation rates. Nor has the 2012 London Olympics left a mass sport participation legacy.

There is UK evidence that individuals who remain active in several sports throughout their late-teens and into their mid-20s are most likely to remain sport participants throughout young adulthood (Roberts et al, 1991). However, there is also UK evidence that the chances of youth sport careers being extended into later life stages are fixed much earlier, in childhood, in sports-active families (Haycock and Smith, 2016; Parry, 2015; Quarmby and Dagkas, 2010; Smith et al, 2015).The most plausible explanation of the relatively high rates of sport participation at all life stages in Nordic countries lies in the norm of childhood socialisation in sportsactive households (Green et al, 2015a; 2015b). There is evidence from the South Caucasus that differences in sport participation rates between socio-demographic groups are fixed by, then remain stable after, age 16 (Birchwood et al, 2008).

The following passages present evidence on how much of this applies in South and East Mediterranean countries which are culturally different, and where modern sports have shallower and briefer historical roots, than in the European and North American societies where most existing evidence has been sourced. Also, we are able to assess whether profiles and predictors of youth careers in sport are also found in other uses of free time in the South and East Mediterranean. In Euro-America we know that certain cultural tastes, specifically in high culture, are unlikely to be acquired after childhood (Hantrais and Kamphorst, 1987; Nagel, 2010). We have evidence from the South and East Mediterranean on youth and young adulthood careers in a much wider range of free time activities than in any previous studies in any part of the world. As well as showing whether the South 
and East Mediterranean countries are similar or different to Western societies in their social demographies of sport participation, we can also see whether local social profiles and trends with age in sport participation are found in a wider range of free time activities in the predominantly Arab and Islamic South and East Mediterranean.

The significance for sports policy concerns whether boosting enduring participation needs sports-specific measures, or whether it may be necessary to change more widely rooted features of the age group's lives. We will then better understand why boosting young people's levels of participation in sports, or widening the range of their sporting repertoires alone, may fail to feed through into higher rates of sport participation in young and later adulthood, and why it may be necessary to try something different, and what kind of difference may prove most efficacious.

\section{Evidence}

This is from interview surveys in 2015-16 among nationally representative samples of around 2000 15-29 year olds in each of five South and East Mediterranean countries (Algeria, Egypt, Lebanon, Morocco and Tunisia). The fieldwork was supervised by local academic partners and conducted by survey organisations with experience of identifying nationally representative samples in their respective countries. The initial samples were always households, in which all residents aged 15-29 were interviewed. Fieldworkers went out in pairs and the interviews were always conducted by a same-sex interviewer. There was complementary qualitative fieldwork in three contrasting locations in each of the five countries (see Roberts et al, 2018c), but the following analysis uses data solely from the quantitative surveys.

The research was planned in the immediate aftermath of the 'Arab Spring' of 2011 when there was international interest in the conditions, grievances and aspirations of the region's youth, and the interviews covered all aspects of respondents' lives - their childhood families, education, employment and unemployment, profiles of religious and political orientations and activities, plus uses of free time for which respondents were asked about their frequency of participation in 16 different activities. Answers were recorded on a six-point scale with a range from never to every day. Throughout the following analysis we use 'at least once a week' as the cut-off for distinguishing regular participants from others. Respondents were also asked to estimate the time that they spent each day watching television and online. We restrict our analysis to single respondents having shown elsewhere that marriage was associated with a general reduction in out-of-home free time activities by men and women (Roberts et al, 2018a). Thus our comparisons between participation rates in different age groups (15-19, 20-24 and 25-29) are assumed to be age effects, features of normal (in the countries) agerelated changes in uses of free time among unmarried youth and young adults, rather than consequences of changes in family circumstances.

Throughout our analysis we divide respondents into age groups and by gender, and also by 'social class' origins, indicated most appropriately for our 
purposes by fathers' education. Occupation proves a less precise indicator in countries where around a third of all male employment is self-employment which can mean anything from subsistence farming to a substantial enterprise. Father's education was a good predictor of whether the fathers held (or had held if subsequently retired) 'salariat' (non-manual) positions. It is also likely to be the better indicator of a household's cultural character. Fathers' and mothers' education were positively inter-related, but fathers were the better educated: 10 percent of fathers against six percent of respondents' mothers had been to university. Most mothers had 'housewife' as their sole occupation. As we had expected, fathers' education predicted their children's educational attainments and thereby indirectly, but also directly, their subsequent employment (Kovacheva et al, 2018; Roberts et al, 2018b).

We are not seeking to explain gender, age or social class differences in uses of free time, but to test whether differences between socio-demographic groups formed by age 15-19 remained unchanged throughout the 20-29 age range, and whether by 15-19 participation rates had peaked and rose no higher. We also test whether respondents' post-16 experiences had independent effects on free time uses. For this purpose we group just the 25-29 age group into those with and without higher education qualifications, and also by their labour force positions. Here we treat males and females differently. Fifty-five percent of all the young adult women in the surveys had never entered the labour market and this 'inactive group' are separated from those who were at least seeking jobs. Males are grouped according to the quality of their employment. Those with 'salariat' jobs (with written contracts of indefinite duration), or who were in informal jobs or selfemployed with incomes above the lowest quartiles in their countries, are separated from the more numerous respondents who were part of a precariat of lower-paid employees, or were currently unemployed, and others who had become long-term inactive in the labour market (see Roberts et al, 2018b).

In addition to sport participation, we present evidence on five additional uses of free time. Two are social uses. One is going out with friends which, especially for males, was the most common free time activity, where we know that the most revealing differences were in where the friends met and exactly what they did (see Roberts et al, 2018c), but there were also differences in whether the various sociodemographic groups went out with friends to do anything at all in a typical week. The second social use of free time is visiting tea rooms and cafes. Then we present evidence on three cultural activities; attending music concerts and/or festivals, visiting the theatre, and visiting libraries. Here our interest lies in whether participation profiles and trends with age are similar or different from those in sport.

Our evidence is cross-sectional. We are not presenting prospective or retrospective longitudinal data. We cannot say how the uses of free time of the individuals in our surveys changed as they grew older. Our measurements are of the propensity to participate within different socio-demographic groups. These may remain stable while many individuals alter their behaviour in ways that cancel out each other's changes. 
Findings

Sport

Table 1a presents the findings from the entire sample of single respondents. The declining Ns in successive age groups, and the lower Ns among females, are due to variations in the proportions of all respondents who were single. In all the directly comparable cells in Table 1a, males have higher sport participation rates than females, and the difference at age 15-19 (21 percent) is very similar to the difference at age 25-29 (18 percent). In all age groups respondents with bettereducated fathers had the higher sport participation rates. Among females the social class gap remained stable across the age groups, whereas among males it widened with age from 14 percent to 19 percent. This was due to highly educated fathers tending to have sons whose sport participation proved enduring from late-teens to late-20s. The sport careers of sons with less educated fathers proved more vulnerable as they progressed through youth and into young adulthood. Daughters of highly educated fathers were also different from other females in that 30 percent were participating in sport at age 15-19 and almost as many, 29 percent, at age 2529. In all the other fathers' education groups, the clear trend with age in sport participation was downward. Males and females with highly educated fathers not only had the highest sport participation rates in all age groups, but were also different in their greater tendency to stick in sport through youth and into young adulthood.

Table 1b shows that 25-29 year olds who were higher education graduates had higher sport participation rates than other members of their age group. This applied among males and females. However, this positive cross-sectional relationship between higher education and sport participation was entirely due to the children of highly educated fathers being over-represented among the young adults who themselves had become higher education graduates. Our evidence does not suggest that higher education was having any independent and consistent effect on levels of sport participation.

The numbers of cases in all the tables that deal only with the 25-29 age group are much smaller than, for example, in Table 1a. This is because the 25-29 year olds were the more likely to be married (not single), then when divided by whether they were higher education graduates (Table 1b) and by their labour market experience or inexperience (Table 1c) as well as by gender and fathers' education, the numbers shrink still further.

Table 1a. All Respondents: Percentages playing Sport at least once a week

\begin{tabular}{|l|c|c|c|c|c|c|c|}
\hline & $\begin{array}{c}\mathbf{1 5 - 1 9} \\
\mathbf{\%}\end{array}$ & $\begin{array}{c}\mathbf{2 0 - 2 4} \\
\mathbf{\%}\end{array}$ & $\begin{array}{c}\mathbf{2 5 - 2 9} \\
\mathbf{\%}\end{array}$ & $\begin{array}{c}\mathbf{1 5 - 1 9} \\
\mathbf{\%}\end{array}$ & $\begin{array}{c}\mathbf{2 0 - 2 4} \\
\mathbf{\%}\end{array}$ & $\begin{array}{c}\mathbf{2 5 - 2 9} \\
\mathbf{\%}\end{array}$ \\
\hline $\begin{array}{l}\text { Father's education: } \\
\text { primary or less }\end{array}$ & 32 & 27 & 25 & 11 & 10 & 8 \\
\hline Middle or upper secondary & 45 & 46 & 31 & 21 & 21 & 10 \\
\hline Higher & 46 & 40 & 44 & & 30 & 19 & 29 \\
\hline All & 39 & 35 & 29 & & 18 & 15 & 11 \\
\hline $\mathrm{N}=$ & 1518 & 1450 & 1043 & & 1208 & 926 & 628 \\
\hline
\end{tabular}


Table 1b. 25-29 year olds only

\begin{tabular}{|l|c|c|c|c|}
\hline \multicolumn{1}{|c|}{ Males } & $\begin{array}{c}\text { Higher } \\
\text { education } \\
\mathbf{\%}\end{array}$ & $\begin{array}{c}\text { Other } \\
\mathbf{\%}\end{array}$ & $\begin{array}{c}\text { Females } \\
\text { edigher } \\
\text { edion }\end{array}$ & $\begin{array}{c}\text { Other } \\
\mathbf{\%}\end{array}$ \\
\hline $\begin{array}{l}\text { Father's education: primary } \\
\text { or less }\end{array}$ & 19 & 24 & 11 & 7 \\
\hline Middle or upper secondary & 34 & 26 & 11 & 8 \\
\hline Higher & 42 & 45 & 21 & 35 \\
\hline All & 31 & 25 & 13 & 9 \\
\hline $\mathrm{N}=$ & 196 & 642 & 163 & 342 \\
\hline
\end{tabular}

Table 1c. 25-29 year olds only Respondent's Occupational Class

\begin{tabular}{|l|c|c|c|c|c|}
\hline & $\begin{array}{c}\text { Musiness or } \\
\text { sub-business, } \\
\text { salariat or } \\
\text { sub-salariat, } \\
\text { informals } \\
\text { \% }\end{array}$ & $\begin{array}{c}\text { Other } \\
\text { (precariat, } \\
\text { inactive, } \\
\text { detached) } \\
\text { \% }\end{array}$ & $\begin{array}{c}\text { Any class } \\
\text { in labour } \\
\text { force } \\
\text { \% }\end{array}$ & $\begin{array}{c}\text { Inactive } \\
\text { \% }\end{array}$ \\
\hline $\begin{array}{l}\text { Father's education: } \\
\text { primary or less }\end{array}$ & 30 & 21 & 26 & 23 \\
\hline $\begin{array}{l}\text { Middle or upper } \\
\text { secondary }\end{array}$ & 26 & 34 & 26 & 43 \\
\hline Higher & 43 & 36 & 42 & 35 \\
\hline All & 29 & 26 & 27 & 29 \\
\hline $\mathrm{N}=$ & 173 & 369 & 287 & 255 \\
\hline
\end{tabular}

This is of greatest concern with the participation rates of respondents with highly educated fathers (just 10 percent of all respondents in the age group). The participation rates in this sub-population (in Tables $1 \mathrm{~b}$ and 1c) are calculated from less than 20 cases. Our response is to attach significance only to trends common in all three fathers' education groups, and when one such group is different, to attach significance to this only if the same difference appears in other free time activities, not to any solitary statistic.

Applying this 'rule', we can see in Table 1c that workforce experience did not have any independent and consistent effects on sport participation rates. Neither higher education nor labour force experiences were altering or even modifying the main variations and stabilities in participation rates by age, gender and class origins. The broad social demography of sport participation appeared to have been set enduringly earlier in the samples' lives.

\section{Social uses of Free Time}

Going out

There were similarities, but also major differences, between the social demography and trends with age in sport participation and social uses of free time. Spending free time with friends was by far the respondents' most common out-of- 
home free time activity. As with sport, in all the directly comparable cells in Table 2a, males were more likely than females to go out with friends at least once a week. Among males, participation fluctuated across the age groups only between 73 percent and 77 percent, whereas the female range was from 36 percent to 48 percent, and the trend with age was downward. Fathers' education (our indicator of social class origins) made no difference to males' participation rates, whereas there was a strong positive association among the young women: those with highly educated fathers were by far the most likely to go out with friends at least once a week.

Table 2a. All Respondents: Percentages going out with Friends at least once a week

\begin{tabular}{|l|c|c|c|c|c|c|c|}
\multicolumn{1}{c}{} & \multicolumn{3}{c|}{ Males } & \multicolumn{1}{c|}{ Females } \\
\hline & $\begin{array}{c}\mathbf{1 5 - 1 9} \\
\mathbf{\%}\end{array}$ & $\begin{array}{c}\mathbf{2 0 - 2 4} \\
\mathbf{\%}\end{array}$ & $\begin{array}{c}\mathbf{2 5 - 2 9} \\
\mathbf{\%}\end{array}$ & $\begin{array}{c}\mathbf{1 5 - 1 9} \\
\mathbf{\%}\end{array}$ & $\begin{array}{c}\mathbf{2 0 - 2 4} \\
\mathbf{\%}\end{array}$ & $\begin{array}{c}\mathbf{2 5 - 2 9} \\
\mathbf{\%}\end{array}$ \\
\hline $\begin{array}{l}\text { Father's education: } \\
\text { primary or less }\end{array}$ & 67 & 70 & 76 & 38 & 32 & 31 \\
\hline Middle or upper secondary & 77 & 78 & 76 & 54 & 53 & 43 \\
\hline Higher & 78 & 76 & 86 & & 57 & 62 & 43 \\
\hline All & 73 & 74 & 77 & 48 & 43 & 36 \\
\hline $\mathrm{N}=$ & 1518 & 1450 & 1043 & & 1208 & 926 & 628 \\
\hline
\end{tabular}

Table 2b. 25-29 year olds only

\begin{tabular}{|l|c|c|c|c|}
\multicolumn{2}{c}{} & \multicolumn{2}{c|}{ Memales } \\
\hline & $\begin{array}{c}\text { Higher } \\
\text { education } \\
\mathbf{\%}\end{array}$ & $\begin{array}{c}\text { Other } \\
\mathbf{\%}\end{array}$ & $\begin{array}{c}\text { Higher } \\
\text { education } \\
\mathbf{\%}\end{array}$ & $\begin{array}{c}\text { Other } \\
\mathbf{\%}\end{array}$ \\
\hline Father's education: primary or less & 77 & 73 & 36 & 33 \\
\hline Middle or upper secondary & 76 & 72 & 50 & 40 \\
\hline Higher & 87 & 83 & 50 & 35 \\
\hline All & 79 & 73 & 44 & 36 \\
\hline N & 196 & 642 & 163 & 342 \\
\hline
\end{tabular}

Table 2c. 25-29 year olds only Respondent's Occupational Class

\begin{tabular}{|l|c|c|c|c|}
\hline & $\begin{array}{c}\text { Business or } \\
\text { sub- } \\
\text { business, } \\
\text { salariat or } \\
\text { sub-salariat, } \\
\text { informals } \\
\text { \% }\end{array}$ & $\begin{array}{c}\text { Mther } \\
\text { (precariat, } \\
\text { inactive, } \\
\text { detached) } \\
\text { \% }\end{array}$ & $\begin{array}{c}\text { Any class } \\
\text { in labour } \\
\text { force } \\
\text { \% }\end{array}$ & $\begin{array}{c}\text { Females } \\
\text { Inactive } \\
\%\end{array}$ \\
\hline $\begin{array}{l}\text { Father's education: } \\
\text { primary or less }\end{array}$ & 72 & 80 & 76 & 79 \\
\hline $\begin{array}{l}\text { Middle or upper } \\
\text { secondary }\end{array}$ & 72 & 85 & 75 & 85 \\
\hline Higher & 87 & 79 & 89 & 70 \\
\hline All & 73 & 81 & 76 & 80 \\
\hline $\mathrm{N}=$ & 173 & 369 & 287 & 255 \\
\hline
\end{tabular}


Higher education was making only a slight difference to males' involvement in this use of free time, but it boosted participation among young women, especially those with the most highly educated fathers (Table $2 b$ ). Positions inside and outside the workforce were making no consistent differences across fathers' education groups among either the males or females (Table 2c).

\section{Cafes and tearooms}

Visiting cafes and tearooms, our second social use of free time, was also second only to going out with friends in the overall participation rates in the various free time activities.

Once again, males out-performed females in all the directly comparable cells in Table 3a. Males' likelihood of visiting a café or tearoom at least once a week rose steadily with age among those whose fathers had ended their education at all levels, but among females this was the case only among those with the most highly educated fathers. A highly educated father was boosting participation rates most strongly among both males and females in our oldest, 25-29 age group (Table 3a).

Male and female respondents aged 25-29 who had graduated from higher education had higher participation rates in visiting cafes and tearooms than others in their age group, and this education effect was strongest among the females, especially among the females with the better-educated fathers (Table 3b). In contrast, the 25-29 year olds' positions inside or outside the workforce were making no consistent differences across the gender and fathers' education groups to respondents' likelihood of visiting cafes or tearooms at least weekly (see Table 3c).

Table 3a. All Respondents: Percentages visiting Cafes or Tearooms at least once a Week

\begin{tabular}{|l|c|c|c|c|c|c|c|}
\multicolumn{4}{c}{ Males } & \multicolumn{1}{c|}{ Females } \\
\hline & $\begin{array}{c}\mathbf{1 5 - 1 9} \\
\mathbf{\%}\end{array}$ & $\begin{array}{c}\mathbf{2 0 - 2 4} \\
\mathbf{\%}\end{array}$ & $\begin{array}{c}\mathbf{2 5 - 2 9} \\
\mathbf{\%}\end{array}$ & $\begin{array}{c}\mathbf{1 5 - 1 9} \\
\mathbf{\%}\end{array}$ & $\begin{array}{c}\mathbf{2 0 - 2 4} \\
\mathbf{\%}\end{array}$ & $\begin{array}{c}\mathbf{2 5 - 2 9} \\
\mathbf{\%}\end{array}$ \\
\hline $\begin{array}{l}\text { Father's education: } \\
\text { primary or less }\end{array}$ & 41 & 51 & 58 & 12 & 11 & 15 \\
\hline Middle or upper secondary & 49 & 57 & 62 & 22 & 26 & 24 \\
\hline Higher & 47 & 53 & 73 & & 24 & 28 & 33 \\
\hline All & 45 & 54 & 61 & & 18 & 18 & 20 \\
\hline $\mathrm{N}=$ & 1518 & 1450 & 1043 & & 1208 & 926 & 628 \\
\hline
\end{tabular}

Table 3b. 25-29 year olds only

\begin{tabular}{|l|c|c|c|c|c|}
\hline \multicolumn{1}{|c|}{ Males } & $\begin{array}{c}\text { Higher } \\
\text { education } \\
\boldsymbol{\%}\end{array}$ & $\begin{array}{c}\text { Other } \\
\boldsymbol{\%}\end{array}$ & $\begin{array}{c}\text { Higher } \\
\text { education } \\
\mathbf{\%}\end{array}$ & $\begin{array}{c}\text { Other } \\
\mathbf{\%}\end{array}$ \\
\hline Father's education: primary or less & 61 & 56 & & 16 & 16 \\
\hline Middle or upper secondary & 61 & 57 & & 34 & 21 \\
\hline Higher & 76 & 66 & & 46 & 26 \\
\hline All & 67 & 57 & & 28 & 18 \\
\hline $\mathrm{N}=$ & 196 & 642 & & 163 & 342 \\
\hline
\end{tabular}


Table 3c. 25-29 year olds only Respondent's Occupational Class

\begin{tabular}{|l|c|c|c|c|}
\hline & $\begin{array}{c}\text { Business or } \\
\text { sub-business, } \\
\text { salariat or } \\
\text { sub-salariat, } \\
\text { informals } \\
\text { \% }\end{array}$ & $\begin{array}{c}\text { Other } \\
\text { (precariat, } \\
\text { inactive, } \\
\text { detached) } \\
\text { \% }\end{array}$ & $\begin{array}{c}\text { Any class } \\
\text { in labour } \\
\text { force } \\
\%\end{array}$ & $\begin{array}{c}\text { Inactive } \\
\%\end{array}$ \\
\hline $\begin{array}{l}\text { Father's education: } \\
\text { primary or less }\end{array}$ & 56 & 64 & 16 & 13 \\
\hline $\begin{array}{l}\text { Middle or upper } \\
\text { secondary }\end{array}$ & 55 & 73 & 28 & 17 \\
\hline Higher & 76 & 71 & 25 & 38 \\
\hline All & 58 & 67 & 22 & 16 \\
\hline $\mathrm{N}=$ & 173 & 369 & 287 & 255 \\
\hline
\end{tabular}

\section{Cultural Activities}

\section{Musical events}

Attendance at these events declined with age, but among males this was only in our oldest, 25-29 years old age group (Table 4a). Males were more likely than females to attend these events, but only marginally so. Participation was related to family class origins, but there were no clear associations with progressing through higher education or respondents' own experiences in the labour market (Tables $4 \mathrm{~b}$ and $4 \mathrm{c})$.

Table 4a. All Respondents: Percentages attending Music Concerts or Festivals at least once a week

\begin{tabular}{|l|c|c|c|c|c|c|c|}
\multicolumn{1}{c}{ Males } & \multicolumn{1}{c|}{ Females } \\
\hline & $\begin{array}{c}\mathbf{1 5 - 1 9} \\
\mathbf{\%}\end{array}$ & $\begin{array}{c}\mathbf{2 0 - 2 4} \\
\mathbf{\%}\end{array}$ & $\begin{array}{c}\mathbf{2 5 - 2 9} \\
\mathbf{\%}\end{array}$ & $\begin{array}{c}\mathbf{1 5 - 1 9} \\
\mathbf{\%}\end{array}$ & $\begin{array}{c}\mathbf{2 0 - 2 4} \\
\mathbf{\%}\end{array}$ & $\begin{array}{c}\mathbf{2 5 - 2 9} \\
\mathbf{\%}\end{array}$ \\
\hline $\begin{array}{l}\text { Father's education: } \\
\text { primary or less }\end{array}$ & 7 & 9 & 6 & 7 & 5 & 3 \\
\hline Middle or upper secondary & 13 & 10 & 6 & & 9 & 8 & 5 \\
\hline Higher & 12 & 12 & 13 & & 12 & 4 & 8 \\
\hline All & 10 & 10 & 7 & & 9 & 6 & 4 \\
\hline N = & 1518 & 1450 & 1043 & & 1208 & 926 & 628 \\
\hline
\end{tabular}

Table 4b. 25-29 year olds only

\begin{tabular}{|l|c|c|c|c|c|}
\multicolumn{2}{c|}{} & \multicolumn{2}{c}{ Males } & \multicolumn{3}{c|}{ Higher } \\
& $\begin{array}{c}\text { education } \\
\mathbf{\%}\end{array}$ & $\begin{array}{c}\text { Other } \\
\mathbf{\%}\end{array}$ & $\begin{array}{c}\text { Higher } \\
\text { education } \\
\mathbf{\%}\end{array}$ & $\begin{array}{c}\text { Other } \\
\%\end{array}$ \\
\hline $\begin{array}{l}\text { Father's education: primary or } \\
\text { less }\end{array}$ & 5 & 7 & & 4 & 2 \\
\hline Middle or upper secondary & 8 & 6 & & 5 & 5 \\
\hline Higher & 7 & 17 & & 0 & 22 \\
\hline All & 7 & 7 & & 4 & 4 \\
\hline $\mathrm{N}=$ & 196 & 642 & & 163 & 342 \\
\hline
\end{tabular}


Table 4c. 25-29 year olds only Respondent's Occupational Class

\begin{tabular}{|l|c|c|c|c|c|}
\hline & \multicolumn{2}{c}{ Males } & \multicolumn{2}{c|}{ Females } \\
\hline & $\begin{array}{c}\text { Business or sub- } \\
\text { business, salariat } \\
\text { or sub-salariat, } \\
\text { informals } \\
\text { \% }\end{array}$ & $\begin{array}{c}\text { Other } \\
\text { (precariat, } \\
\text { inactive, } \\
\text { detached) } \\
\text { \% }\end{array}$ & $\begin{array}{c}\text { Any class in } \\
\text { labour force } \\
\%\end{array}$ & $\begin{array}{c}\text { Inactive } \\
\%\end{array}$ \\
\hline $\begin{array}{l}\text { Father's education: } \\
\text { primary or less }\end{array}$ & 7 & 5 & 6 & 5 \\
\hline $\begin{array}{l}\text { Middle or upper } \\
\text { secondary }\end{array}$ & 5 & 5 & 4 & 9 \\
\hline Higher & 11 & 7 & 9 & 10 \\
\hline All & 6 & 5 & & 3 & 6 \\
\hline $\mathrm{N}=$ & 173 & 369 & 287 & 255 \\
\hline
\end{tabular}

Theatre

Here, unlike any other free time activity that we feature, attendances peaked in the 20-24 age group then dropped to their lowest level among the 25-29 year olds. Family class origins were making a difference, but their association with participation rates began only after age 20 . Once again, males were more involved than females (Table 5a). Also once again, there were no clear associations with whether respondents had progressed through higher education or their positions in the workforce (Tables $5 \mathrm{~b}$ and $5 \mathrm{c}$ ).

Table 5a. All Respondents: Percentages visiting a Theatre at least once a week

\begin{tabular}{|l|c|c|c|c|c|c|}
\multicolumn{1}{|c|}{ Males } \\
\hline $\begin{array}{c}\mathbf{1 5 - 1 9} \\
\mathbf{\%}\end{array}$ & $\begin{array}{c}\mathbf{2 0 - 2 4} \\
\mathbf{\%}\end{array}$ & $\begin{array}{c}\mathbf{2 5 - 2 9} \\
\mathbf{\%}\end{array}$ & $\begin{array}{c}\mathbf{1 5 - 1 9} \\
\mathbf{\%}\end{array}$ & $\begin{array}{c}\mathbf{2 0 - 2 4} \\
\mathbf{\%}\end{array}$ & $\begin{array}{c}\mathbf{2 5 - 2 9} \\
\mathbf{\%}\end{array}$ \\
\hline $\begin{array}{l}\text { Father's education: } \\
\text { primary or less }\end{array}$ & 5 & 7 & 3 & 3 & 3 & 1 \\
\hline $\begin{array}{l}\text { Middle or upper } \\
\text { secondary }\end{array}$ & 5 & 7 & 3 & 3 & 4 & 1 \\
\hline Higher & 3 & 11 & 6 & 2 & 9 & 3 \\
\hline All & 5 & 7 & 3 & 3 & 4 & 1 \\
\hline $\mathrm{N}=$ & 1518 & 1450 & 1043 & 1208 & 926 & 628 \\
\hline
\end{tabular}

Table 5b. 25-29 year olds only

\begin{tabular}{|l|c|c|c|c|c|}
\multicolumn{1}{c}{} & \multicolumn{2}{c|}{ Males } & \multicolumn{3}{c|}{ Females } \\
\hline & $\begin{array}{c}\text { Higher } \\
\text { education } \\
\mathbf{\%}\end{array}$ & $\begin{array}{c}\text { Other } \\
\mathbf{\%}\end{array}$ & $\begin{array}{c}\text { Higher } \\
\text { education } \\
\mathbf{\%}\end{array}$ & $\begin{array}{c}\text { Other } \\
\mathbf{\%}\end{array}$ \\
\hline Father's education: primary or less & 2 & 4 & & 1 & 2 \\
\hline Middle or upper secondary & 1 & 3 & & 3 & 0 \\
\hline Higher & 7 & 17 & & 2 & 10 \\
\hline All & 2 & 4 & & 2 & 2 \\
\hline N & 196 & 642 & & 163 & 342 \\
\hline
\end{tabular}


Table 5c. 25-29 year olds only Respondent's Occupational Class

Males

\begin{tabular}{|l|c|c|c|c|}
\hline & $\begin{array}{c}\text { Business or } \\
\text { sub- } \\
\text { business, } \\
\text { salariat or } \\
\text { sub-salariat, } \\
\text { informals } \\
\text { \% }\end{array}$ & $\begin{array}{c}\text { Other } \\
\text { (precariat, } \\
\text { inactive, } \\
\text { detached) } \\
\mathbf{\%}\end{array}$ & $\begin{array}{c}\text { Any class } \\
\text { in labour } \\
\text { force } \\
\%\end{array}$ & $\begin{array}{c}\text { Inactive } \\
\%\end{array}$ \\
\hline $\begin{array}{l}\text { Father's education: } \\
\text { primary or less }\end{array}$ & 3 & 2 & 3 & 1 \\
\hline $\begin{array}{l}\text { Middle or upper } \\
\text { secondary }\end{array}$ & 2 & 2 & 2 & 3 \\
\hline Higher & 11 & 7 & 2 & 4 \\
\hline All & 2 & 2 & 2 & 2 \\
\hline $\mathrm{N}=$ & 173 & 369 & 287 & 255 \\
\hline
\end{tabular}

\section{$\underline{\text { Libraries }}$}

This is the sole free time activity among those featured here where there was no sign of a gender effect. Otherwise, as with sport, participation declined with age, and family class origins were making a difference in all gender/age groups (Table 6a). Respondents' own labour market experiences were making no difference to their likelihood of visiting libraries (Table 6c). Nor was higher education among the females, except that when males and females with highly educated fathers had not themselves progressed through higher education, this appeared to have boosted their propensity to visit (Table $6 \mathrm{~b}$ ).

Table 6a. All Respondents: Percentages visiting a Library at least once a week

\begin{tabular}{|l|c|c|c|c|c|c|c|}
\multicolumn{1}{c}{ Males } & $\begin{array}{c}\mathbf{1 5 - 1 9} \\
\mathbf{\%}\end{array}$ & $\begin{array}{c}\mathbf{2 0 - 2 4} \\
\mathbf{\%}\end{array}$ & $\begin{array}{c}\mathbf{2 5 - 2 9} \\
\mathbf{\%}\end{array}$ & $\begin{array}{c}\mathbf{1 5 - 1 9} \\
\mathbf{\%}\end{array}$ & $\begin{array}{c}\mathbf{2 0 - 2 4} \\
\mathbf{\%}\end{array}$ & $\begin{array}{c}\mathbf{2 5 - 2 9} \\
\mathbf{\%}\end{array}$ \\
\hline $\begin{array}{l}\text { Father's education: } \\
\text { primary or less }\end{array}$ & 13 & 13 & 7 & & 18 & 15 & 7 \\
\hline $\begin{array}{l}\text { Middle or upper } \\
\text { secondary }\end{array}$ & 19 & 16 & 8 & & 23 & 19 & 8 \\
\hline Higher & 27 & 26 & 19 & & 28 & 22 & 14 \\
\hline All & 17 & 15 & 8 & & 22 & 17 & 8 \\
\hline $\mathrm{N}=$ & 1518 & 1450 & 1043 & & 1208 & 926 & 628 \\
\hline
\end{tabular}

Table 6b. 25-29 year olds only

\begin{tabular}{|l|c|c|c|c|c|}
\multicolumn{2}{c}{} & \multicolumn{2}{c}{ Males } & \multicolumn{2}{c|}{ Females } \\
\hline & $\begin{array}{c}\text { Higher } \\
\text { education } \\
\mathbf{\%}\end{array}$ & $\begin{array}{c}\text { Other } \\
\mathbf{\%}\end{array}$ & $\begin{array}{c}\text { Higher } \\
\text { education } \\
\mathbf{\%}\end{array}$ & $\begin{array}{c}\text { Other } \\
\boldsymbol{\%}\end{array}$ \\
\hline Father's education: primary or less & 6 & 8 & 7 & 7 \\
\hline Middle or upper secondary & 6 & 10 & 13 & 6 \\
\hline Higher & 11 & 31 & 0 & 35 \\
\hline All & 7 & 10 & 8 & 9 \\
\hline $\mathrm{N}=$ & 196 & 642 & 163 & 342 \\
\hline
\end{tabular}


Table 6c. 25-29 year olds only Respondent's Occupational Class

\begin{tabular}{|l|c|c|c|c|}
\hline & $\begin{array}{c}\text { Business or } \\
\text { sub- } \\
\text { business, } \\
\text { salariat or } \\
\text { sub-salariat, } \\
\text { informals } \\
\text { \% }\end{array}$ & $\begin{array}{c}\text { Other } \\
\text { (precariat, } \\
\text { inactive, } \\
\text { detached) } \\
\text { \% }\end{array}$ & $\begin{array}{c}\text { Any class } \\
\text { in labour } \\
\text { force } \\
\%\end{array}$ & $\begin{array}{c}\text { Inactive } \\
\%\end{array}$ \\
\hline $\begin{array}{l}\text { Father's education: } \\
\text { primary or less }\end{array}$ & 6 & 3 & 5 & 2 \\
\hline $\begin{array}{l}\text { Middle or upper } \\
\text { secondary }\end{array}$ & 3 & 9 & 5 & 10 \\
\hline Higher & 14 & 7 & 11 & 10 \\
\hline All & 6 & 5 & 6 & 6 \\
\hline $\mathrm{N}=$ & 173 & 369 & 287 & 255 \\
\hline
\end{tabular}

\section{Discussion and Conclusions}

\section{Discussion}

The cultural free time activities that we have examined did not exhibit similar socio-demographic participation profiles or trends with age during youth and young adulthood. Library visiting was different from all the other activities (sports, social and cultural) in the complete absence of any gender effect. Theatre visits were also unique among the activities being considered here in that 20-24 was the age when attendances peaked. However, a very distinctive feature of the entire cultural sector is that gender differences, if present at all, were far narrower than in sport or social uses of free time. In contrast, all three cultural activities were similar to sport in the clear and consistent relationships across age and gender groups with family class origins, indicated by fathers' education. With family class origins controlled, there was never a clear relationship between participation in sport or any of the cultural activities on the one side, then on the other, whether respondents had progressed through higher education or their current positions inside or outside the labour market. An important exception was that in the cultural activities, respondents with highly educated fathers who had not themselves progressed through higher education appeared to be compensating with exceptionally high rates of participation in all three cultural activities. The obverse may also have applied: university graduates whose fathers were equally educated may have felt no need to add further cultural distinction to their social identities (Bourdieu, 1984). It was not just the cultural, but rather every single free time activity that we have examined that displayed distinctive socio-demographic features. Social uses of free time differed from one another as well as displaying overall differences from cultural and sports activities. There were neither differences by age nor fathers' education in 'going out' rates among the males. 
Visits to cafes and tearooms rose with age among the males. These findings were not replicated in any other free time activity.

That said, other socio-demographic features recurred in most free time activities. Males were usually more involved than females. Peak activity was usually at age 15-19 then at best was maintained but more often declined. Respondents with highly educated fathers usually had the highest participation rates, and these relatively high rates were likely to endure throughout their $20 \mathrm{~s}$. The combination of a highly educated father and highly educated son or daughter had distinctive consequences. It boosted female rates of 'going out' and 'visits to cafes and tearooms'. Counter-intuitively, as noted above, sons and daughters of highly educated fathers who themselves had not progressed through higher education were more likely to be involved in all three cultural activities than when both generations were graduates.

Sport proved similar to cultural activities in the strong effects of fathers' education, suggesting that childhood socialisation was making a lasting impression on these uses of free time, and this applied equally to sons and daughters. Family origins appeared to set limits to sport and cultural participation rates which could be exceeded during early youth only to drop back when young adults entered their 20s. However, sport differed from cultural activities in the wider gender differences in the former, and the relatively step downward trend with age except among males with highly educated fathers.

\section{Conclusions}

We now have persuasive evidence from the UK, the South Caucasus, and the South and East Mediterranean, that the foundations for enduring careers in participant sport are usually laid in childhood and early adolescence in specific family contexts. Parents and siblings may be sports-active, or the families may simply encourage their children to play. We can add that in the South and East Mediterranean, and possibly elsewhere, the same families also foster participation in cultural free time activities. This encouragement appears to be part of family cultures in which progression through higher education is treated as just normal. In Europe further progression into salariat careers is also treated as normal, but currently this cannot be so in the South and East Mediterranean because the supply of suitably qualified young people vastly exceeds the number of salariat jobs. Sport participation by older children and young people can probably be boosted in school and community facilities and projects, but all the evidence now available suggests that participation is unlikely to endure into later life stages unless supportive foundations have been laid in childhood family cultures.

Extending the family cultures upon which long term careers in participant sport are built will be difficult given that modern sports were originally designed to be played by boys and young men, and more specifically by those in elite secondary schools and universities. Modern sports were then easily spread into other classes, and all over the world, as forms of exciting entertainment and uses of free time, but it now appears that participation, rather than just entertainment, 
has endured into adulthood only amid supportive family and sport club-based cultures. In the West these supportive families and sport club cultures now embrace girls and women, but up to now any such trend has been far weaker in the South and East Mediterranean.

There is no mechanism whereby family experiences in childhood can mechanistically cause persons aged 20-something to play sport. Causation must be via a propensity to play which is a property of a sub-population. The propensity can keep a participation rate stable while whether any individual plays will depend on a host of other circumstances. The underlying propensity to play can change. It did so when and where modern sports were originally invented, which was at a time when people were moving from rural into urban areas and industrial employment. The main change in Western family free time cultures in the $20^{\text {th }}$ century followed the advent of mass media, especially television. For a few decades television drew families together in front of 'the box in the living room' before bedroom sets, then laptops, tablets and smartphones dispersed viewing into private and other public spaces.

Elite sport (the top events and players in the most popular sports) has developed into a global entertainment business which enriches its star players, promoters and media companies. Much participant sport has moved from playing fields to indoor spaces, and large team sports have been partly replaced by small group games and individual exercise in gyms, parks and along roads. None of these changes have expanded the family cultures in which childhood socialisation makes physically active recreation a normal part of everyday life, for life. The addition of various kinds of theme parks to amusement parks and heritage sites has extended the possibilities for family days-out. Participant sport needs an equivalent wave of entrepreneurialism, probably promoting new forms of sport as family recreation, if it is to contribute to alleviating an obesity crisis. This should become part of the sports research agenda. We now know from experience that simply promoting existing sports among young people more vigorously and extensively through schools and community associations will not yield lifelong changes. It is now time to discover whether new sports can be pioneered and promoted, and make a lifelong enduring difference to participation rates.

\section{References}

Bennett A (2006), 'Punk's not dead: the continuing significance of punk rock for an older generation of fans', Sociology, 40, 219-235.

Bennett A (2013), Music, Style and Ageing: Growing Old Disgracefully, Temple University Press, Philadelphia.

Bennett A and Hodkinson P, eds (2012), Ageing and Youth Cultures, Berg, Oxford.

Birchwood D, Roberts K and Pollock G (2008), 'Explaining differences in sport participation rates among young adults: evidence from the South Caucasus', European Physical Education Review, 14, 283-300.

Bourdieu P (1984), Distinction: A Social Critique of the Judgment of Taste, Routledge, London. 
Department for Culture, Media and Sport (2008), Taking Part: England's Survey of Culture, Leisure and Sport. Headline Findings from the Child Survey 2007, Department for Culture, Media and Sport, London.

Green K, Thurston M and Vaage O (2015a), 'Isn't it good, Norwegian wood? Lifestyle and adventure sports participation among Norwegian youth', Leisure Studies, 34, 529-546.

Green K, Thurston M, Vaage O. and Roberts K. (2015b) “"We're on the right track, baby, we were born that way!" Exploring sports participation in Norway', Sport, Education \& Society, 20, 285-303.

Hantrais L and Kamphorst T J, eds (1987), Trends in the Arts: A Multinational Perspective, Giordano Bruno, Amersfoort.

Haycock D and Smith A (2014), 'A family affair? Exploring the influence of childhood sports socialisation on young adults' leisure sports careers in north-west England', Leisure Studies, 33, 285-304.

Hendry L B (1983), Growing Up and Going Out, Aberdeen University Press, Aberdeen.

Hendry L B, Kloep M, Espnes G A, Ingebrigtsen J E, Glendinning A and Wood S (2002), 'Leisure transitions - a rural perspective', Leisure Studies, 21, 1-14.

Hendry L B, Shucksmith J, Love J G and Glendinning A (1993), Young People's Leisure and Lifestyles, Routledge, London.

Hodkinson P (2002), Goths: Identity, Style and Subculture, Berg, Oxford.

Hodkinson P (2011), 'Ageing in a spectacular "youth culture": continuity, change and community amongst older goths', British Journal of Sociology, 62, 262-282.

Kovacheva S, Roberts K and Kabaivanov S (2018), 'Education to employment transitions in South and East Mediterranean countries', International Journal of Social Science and Economic Research, 3, 2018, 532-559.

MacDonald R and Shildrick T (2007), 'Street corner society: leisure careers, youth (sub)culture and social exclusion', Leisure Studies, 26, 339-355.

Mason V (1995), Young People and Sport: A National Study 1994, Office of Population, Censuses and Surveys, London.

McGuire F A, Dottavio F D and O'Leary J T (1987), 'The relationship of early life experiences to later life leisure involvement', Leisure Sciences, 9, 251-257.

Nagel I (2010), 'Cultural participation between the ages of 14 and 24: intergenerational transmission or cultural mobility?' European Sociological Review, 26, 541-556

Parry W (2015), Do Active Children Become Active Adults? Investigating Experiences of Sport and Exercise Using the 1970 British Cohort Study, PhD thesis, Institute of Education, University College, London.

Quarmby T and Dagkas S (2010), 'Children's engagement in leisure time physical activity: exploring family structure as a determinant', Leisure Studies, 29, 53-66.

Roberts K, Kovacheva S and Kabaivanov S (2018a) 'Leisure and the life-cycle squeeze among young adults in North Africa countries', International Journal of the Sociology of Leisure, 1, 29-42.

Roberts K, Kovacheva S and Kabaivanov S (2018b), 'Class reproduction and re-formation during young people's education to employment transitions in South and East Mediterranean countries', Athens Journal of Mediterranean Studies, 4, 179-200.

Roberts K, Kovacheva S and Kabaivanov S (2018c), 'Uses of free time by young adults in Arab Mediterranean countries: exposing and addressing boundary issues in leisure studies', International Journal of Humanities and Social Science Research, 2018, 4, 10-21,

Roberts K, Minten J H, Chadwick C, Lamb K L and Brodie D A (1991), 'Sporting lives: a case study of leisure careers', Society and Leisure, 14, 261-284. 
Scott D and Willits F K (1989), 'Adolescent and adult leisure patterns: a 37 year follow-up study', Leisure Sciences, 11, 323-335.

Smith L, Gardner B, Aggio D and Hamer M (2015), 'Association between participation in outdoor play and sport at age 10 with physical activity in adulthood', Preventive Medicine. Doi: 10.1016/j.ypmed.2015.02.004.

Sport England (2003), Young People and Sport in England: Trends in Participation 19942002, Sport England, London.

Waechter N and Hollauf I (2018), 'Soziale herausforderungen und entwicklungsaufgaben im medienalltag jugendlicher videospieler/innen', Deutsche Jugend: Zeitschrift für die Jugendarbeit, 5, 218-226 